\title{
Feasibility of Electrochemical Fingerprinting for Plant Phylogeography Study: A Case of Chimonanthus praecox
}

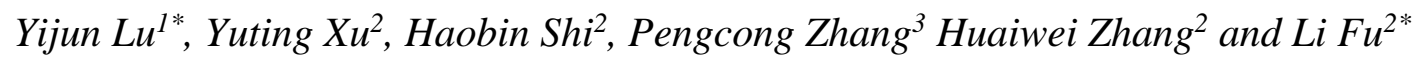 \\ ${ }^{1}$ Artistic Design \& Creation School, Zhejiang University City College, Hangzhou, 310015, P.R. China \\ ${ }^{2}$ College of Materials and Environmental Engineering, Hangzhou Dianzi University, Hangzhou, \\ 310018, P.R. China \\ ${ }^{3}$ Hangzhou Botanical Garden, Hangzhou, Zhejiang, 310013, P.R. China \\ *E-mail: luyijun@zucc.edu.cn, fuli@hdu.edu.cn
}

doi: $10.20964 / 2020.01 .64$

Received: 8 September 2019 / Accepted: 28 October 2019 / Published: 30 November 2019

\begin{abstract}
Investigation of plant relationships is very important in phylogeny and plant culture. In this work, we proposed an electrochemical fingerprint-based method for the investigation of Chimonanthus praecox from different locations. This is the first time that electrochemical fingerprinting technology has been applied to the study of the relationships between subspecies. Three buffer solutions were used as a solvents along with an electrolyte during the Chimonanthus praecox leaf extraction and fingerprint recording. The voltammetric curves of Chimonanthus praecox from different locations exhibited very similar profiles, confirming the feasibility of species identification based on the electrochemical fingerprint. A two-dimensional scatter pattern deduced from two sets of fingerprints was used to distinguish the locations. In addition, this phylogeographic study based on electrochemical fingerprinting suggested that Chimonanthus praecox has multisite and multitime origins.
\end{abstract}

Keywords: Electrochemical fingerprints; Plant identification; Taxonomic sensing; Solid state electrochemistry; Chimonanthus praecox

\section{FULL TEXT}

(C) 2020 The Authors. Published by ESG (www.electrochemsci.org). This article is an open access article distributed under the terms and conditions of the Creative Commons Attribution license (http://creativecommons.org/licenses/by/4.0/). 